\title{
Taxonomy of Trust Relationships in Peer-to-Peer (P2P) Communication
}

\author{
Farookh Khadeer Hussain', Elizabeth Chang ${ }^{1}$, Tharam Dillon ${ }^{2}$ \\ ' School of Information Systems \\ Curtin University of Technology \\ GPO Box U1987, Perth WA 6845, Australia \\ \{HussainF, ChangE\}@cbs . curtin.edu.au \\ ${ }^{2}$ Faculty of IT \\ University of Technology, Sydney \\ Broadway, Sydney, NSW 2007, Australia \\ tharam@it.uts.edu.au
}

\begin{abstract}
Trust between two communicating peers is increasingly catching the attention of the research community. Numerous trust models and trust management protocols have been proposed to enable the task of establishing trust between two communicating peers. In this paper, we enumerate all the possible types of trust relationships between two peers in $\mathrm{P} 2 \mathrm{P}$ communication, with examples. Additionally, we discuss the conditions when a given trust relationship between two peers is feasible.
\end{abstract}

\section{Introduction}

Trust has been analyzed from social and psychological perspectives [5] and has long been a focal point of interpersonal relationships. Many of us use the word TRUST in our daily lives. However, trust has different interpretations and different meanings in different contexts and domains.

In the literature, Marsh was the first person to introduce the concept of trust in computer science [4]. He introduced the notion of trust in distributed artificial intelligence.

In this paper, we focus on the possible trust relationships in peer-to-peer communication. We discuss the conditions under which a given trust relationship is feasible. This paper is organized as follows; Section 1 is brief introduction to trust, in Section 2 we present the various possible trust relationships and Section 3 is the summary of this paper along with future work.

\section{Trust Relationships in Peer-to-Peer (P2P) Communication}

In this section we discuss with examples the various possible trust relationships in peer-to-peer communication. In Section 2.1, we present a formal definition of what 
we mean by a trust relationship. In Section 2.2 we discuss the various possible trust relationships. We believe that the various possible trust relationships in P2P communication are:

1. Implicit Trust Relationship

2. Mutual Trust Relationship

3. Group Trust Relationship

4. Federation Trust Relationship

\subsection{Definition of Trust Relationship}

We define a trust relationship as ' $a$ bond or association between the involved peer/s, which signifies the trust between the involved peer/s'.

In this paper, we use the terms 'person' and 'peer' interchangeable because a person is always behind a peer and a peer's behavior depends directly on the person controlling it.

\subsection{Implicit Trust Relationship}

We define the binding between a person and itself, which signifies the belief in its capability or willingness to perform an action at a given point of time, as 'Implicit Trust Relationship'. We believe that implicit trust relationships depend on the following factors:

- The capability or willingness of the person to perform a specific action at a given point in time; and

- Whether the person is an optimist or a pessimist.

Some peers have the tendency to believe that they can do everything in this world (optimists), while they may not necessarily be capable of doing it. In contrast, some people may have a pessimistic attitude towards things and they tend to underrate their capabilities (pessimists). We call this relationship of a given peer in itself that signifies the trust that it has in itself to perform a specific action at a given point in time as Implicit Trust Relationship. In implicit trust relationships, only one peer is involved. Implicit trust relationships are a subset of all the rest of the trust relationships.

\subsection{Mutual Trust Relationship}

We define the binding between a peer and another peer, which signifies the belief in the other peer's capability or willingness to perform an action at a given point in time, as a 'Mutual Trust Relationship'. This is the most common form of trust relationships in P2P communication. In mutual trust relationships, there are exactly two peers who are bound by the trust relationship. We believe that implicit trust relationships depend on the following factors:

- The capability or willingness of a peer to perform a specific action at a given point in time, as perceived by another peer; 
- The psychological type of the peers involved in the mutual trust relationship $[2,3]$;

- The outcome of the previous interactions between the peers in the mutual trust relationship; and

- The degree of the trust recommended by the intermediate peer [1].

\subsection{Group Trust Relationships}

We define a group of peers as 'a collection of more than two peers who perform a set of coherently related tasks and each peer in the group trusts every other peer in the group, for a given context, at a given point in time'.

We define the binding between a peer and another peer/s, belonging to the same group, which signifies the belief the other peer's capability or willingness to perform an action at a given point in time as 'Group Trust Relationship'.

Unlike mutual trust relationships, group trust relationships can involve two or more than two peers. The peers, however, must belong to the same group which is not the case in mutual trust relationship.

We feel that trust relationship between two peers who belong to the same group is a group trust relationship and not a mutual trust relationship. Additionally, we believe that just like trust, groups are formed for a specific context. Two peers who are members of a group for a specific context may or may not be members of same group for another context.

For example consider the following scenario:

Let us assume that we have a set of peers A1, A2, A3, A4 ....A10. Each peer trusts the other peer for its authenticity at a given point in time. This set of peers A1.....A10 is said to form a group where every peer in the group trusts the authenticity of the other members of the group. The trust relationship between A1 and $A 3$ is an example of group trust relationship and not mutual trust relationship.

If A11, which is a new peer, joins this group and all the members in the group trust Al1 for its authenticity, then A11 becomes a member of the group for that context.

\subsection{Federation Trust Relationships}

For defining 'Federated Trust Relationships', we need to first define and explain what we mean by Federated P2P Communication. We define Federated P2P Communication as the communication that takes place between two or more than two groups of peers.

As we mentioned in Section 2.4, the members in each of these groups are centered on a set of coherent interest/s and the peers belonging to a group perform a specific task. In Federated P2P Communication, a peer acts on behalf of the group to which it belongs. If two peers are communicating with each other, it is analogous to two distinct groups communicating with each other. The communication between the groups of peers is regarded as Federated P2P Communication. 
We define 'Federated Trust Relationships' as the binding between two or more than two distinct groups of peers which signify the peer group's belief in the peer group's capability or willingness to perform an action at a given point in time.

A peer in a federated P2P communication may be a member of more than one group. We strongly believe that this is the way that P2P communication will be organized in the future.

For example, let us consider a peer-to-peer file-sharing application, Gnutella. Users of Gnutella can share files with each other. Users may share files like music files, educational documents, documents related to politics etc. Each of these types of files forms a different domain of interest. These domains of interest can be further subdivided. For example, some users who are members of the group which shares music files may be interested in sharing just English songs and not songs composed in any other language. Similarly, some users who are members of the group that shares political documents may be interested in documents related to a particular country/group. A group, as explained above, is formed by a set of peers who have a coherent interest. A peer can join and leave the group at will. Groups can be formed dynamically and destroyed dynamically. Peers can join any group and leave any group.

Let us assume that there are two groups Group A and Group B as shown below. Let us further assume that Peer A and Peer B belong to Group A and Peer C and Peer $\mathrm{D}$ belong to Group $\mathrm{B}$.

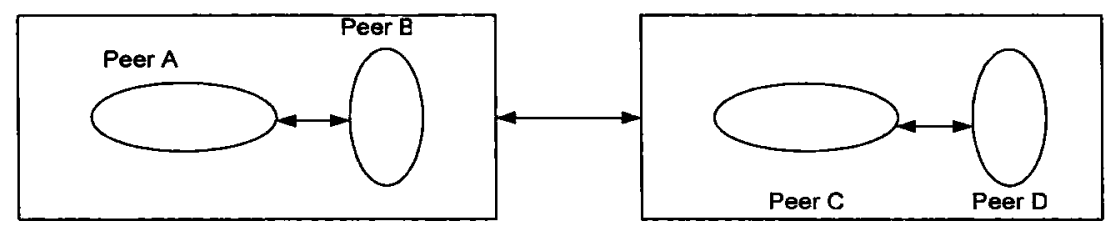

Group A

Group B

Let us assume that Peer A who belongs to Group A, wants to interact with Peer C of Group B. The binding between Peer A and Peer $C$, an example of the federated trust relationships and the communication between them is an example of federated P2P communication

The binding between Peer A and Peer B or the binding between Peer C and Peer D is an example of group trust relationships.

The proposed federated $\mathrm{P} 2 \mathrm{P}$ communication structure has all the features that should be present for a P2P communication. We believe that in the future P2P communication will be organized in this way, because a given document can be found with much ease, with less usage of bandwidth as can be found in non-federated P2P communication. In non-federated $\mathrm{P} 2 \mathrm{P}$ communication, to locate a particular document, the search query is broadcast to the whole network thus resulting in inefficient use of bandwidth. In federated P2P communication, the query is broadcast only to the members of the group thus leading to a far more efficient use of bandwidth. If a peer is a member of more than one group, the query is broadcast to the group, which has the maximum probability of answering the query. We believe 
that federation trust relationships may exist between two, or more than two, groups of peers.

\section{References}

1. Hussain, F.K., Chang, E. \& Dillon,T.S., 'A methodology for reputation management in peer-to-peer (P2P) communication'. (Working Paper)

2. Hussain, F.K., Chang, E. \& Dillon, T.S., 2004, 'Factors of trust that affect trustworthiness in peer-to-peer (P2P) based e-commerce', Proceedings of the International Conference of Business and Information, Taiwan.

3. Hussain, F.K., Chang, E. \& Dillon, T.S., 2004, 'Classification of trust in peer-topeer (P2P) communication', International Journal of Engineering Intelligent Systems, vol. 12.

4. Marsh, S., 1994, 'Formalizing trust as a computational concept', University of Stirling, UK.

5. Smith, J.H., 2002, 'The architectures of trust', Faculty of Humanities, Copenhagen, University of Copenhagen. 